\title{
Study of anatomical changes in Coccinella septempunctata (Coleoptera: Coccinellidae) induced by diet and by infection with the larva of Dinocampus coccinellae (Hymenoptera: Braconidae) using magnetic resonance microimaging
}

\author{
Irene E. GEOGHEGAN ${ }^{*}$, John A. CHUDEK ${ }^{2}$, Regina L. MACKAY ${ }^{2}$, Christian LOWE ${ }^{2}$, Sven MORITZ ${ }^{2}$, Ronald J. \\ MCNICOL $^{1}$, A. Nicholas E. BIRCH ${ }^{1}$, GeOFFrey HUNTER ${ }^{2}$ and MiChaEl E.N. MAJERUS ${ }^{3}$
}

${ }^{1}$ Scottish Crop Research Institute, Invergowrie, Dundee, DD2 5DA, Scotland, UK

${ }^{2}$ Department of Chemistry, University of Dundee, Dundee, DD1 4HN, Scotland, UK

${ }^{3}$ Department of Genetics, University of Cambridge, Cambridge, CB2 3EH, England, UK

Key words. Coccinella septempunctata, Dinocampus coccinellae, parasitisation, diet, nuclear magnetic resonance (NMR), magnetic resonance microimaging (MRM), relaxation measurement, $\mathrm{T}_{1}$ and $\mathrm{T}_{2}$

\begin{abstract}
A range of nuclear magnetic resonance (NMR) techniques, ${ }^{1} \mathrm{H}$ liquid spectroscopy and $\mathrm{T}_{1}$ and $\mathrm{T}_{2}$ relaxation measurements, and microimaging, have been used to observe changes taking place within the bodies of live samples of Coccinella septempunctata, under a variety of conditions. NMR measurements showed that various organs could be seen and identified. It also showed that by changing the diet of the ladybird from aphids [Acyrthosiphon pisum (Harris)] to a standard artificial diet, major changes took place in the insects' tissues. By using a combination of all three techniques it was concluded that on changing the diet of C. septempunctata a mass of nutrient was built up within the insect's abdomen possibly in the same manner as happens before diapause. Changes in the response to NMR measurements were also seen after infection of $C$. septempunctata by the parasitoid wasp (Dinocampus coccinellae). Most significantly an image of the parasitoid larva could be seen within the body mass of the ladybird. It was concluded that NMR could become a major tool in the non-destructive study of insects not just as a means of studying anatomy but also to observe changes in the nature of body tissue.
\end{abstract}

\section{INTRODUCTION}

Predatory coccinellids are considered economically important insects in that they consume vast amounts of plant pests. Consequently, naturally occurring species are worth many millions of pounds every year to agriculturists by reducing aphid damage to crops (Majerus, 1994). Coccinellids have been widely used, with varying degrees of success, as biological control agents. Methods of use have included classical biological control, augmentative biological control and conservation of indigenous predator populations (Luck, 1990; Majerus, 1994). The most important, common British coccinellid in this respect is Coccinella septempunctata (Majerus, 1994). However the lack of success in nature of the coccinellids in controlling pest populations has, in some cases, been attributed to the coccinellid specific parasitoid wasp, Dinocampus coccinellae (Schrank) (Miller, 1971; Majerus, 1994). This braconid wasp exhibits thelytokous parthenogenesis, attacking a wide range, but not all species, of coccinellids (Ceryngier \& Hodek, 1996; Majerus, 1997). It is multivoltine over much of its geographical range (Ceryngier \& Hodek, 1996), including Britain (Majerus, 1997) and especially in Scotland (Geoghegan unpubl. data). In Europe, the generally abundant, C. septempunctata is the most used host of $D$. coccinellae (Iperti, 1964; Klausnitzer, 1969).
Over the past three years (1996-99), levels of parasitisation by this parasitoid have increased dramatically in some Scottish samples of C. septempunctata (Geoghegan et al., 1997) to the detriment of wild population densities of the host (Geoghegan, unpubl. data). This has prompted a more systematic study of the relationship between these two insects.

One technique being used in the investigation is magnetic resonance microimaging which, initial investigations have shown, could become a major tool in this work. Nuclear magnetic resonance microimaging (MRM) along with magnetic resonance imaging (MRI), used widely in hospitals, are developments of nuclear magnetic resonance spectroscopy (NMR). Each imaging technique uses the same principle but, by utilising magnets of considerably greater field strength and smaller bore, MRM is potentially capable of producing image samples to a much higher resolution, pixels or voxels down to between 5 and $10 \mathrm{~mm}$ on a side compared to the $5 \mathrm{~mm}$ typically attained by MRI (Kuhn, 1990). MRM is non-invasive and nondestructive. Its use allows specimens to be re-examined over time or after treatment (for example, in the course of this work, infecting samples). As its name implies, NMR spectroscopy measures the interactions of atomic nuclei with magnetic fields. Different atomic nuclei have different nuclear spins and when a powerful magnetic field is applied these nuclear spins align themselves to the field

\footnotetext{
* Author for correspondence; e-mail: igeogh@scri.sari.ac.uk
} 
in a finite number of allowed orientations. These orientations can be perturbed by applying a force, for example from a burst of radio frequency (rf) energy, after initial absorption of the energy it is retransmitted again as $\mathrm{rf}$. It is this re-transmitted $\mathrm{rf}$ which is detected. Although the nuclei of many isotopes can theoretically be imaged, sensitivity restraints limit the range to a select few of which the hydrogen nucleus $\left({ }^{1} \mathrm{H}\right)$ is by far the most commonly observed. Intra- and intermolecular interactions, along with the strength of the applied magnetic field, determine the frequency at which energy is absorbed. In a uniform field, therefore, moieties of the same type, in equivalent environments, will absorb energy (or resonate) at the same frequency. The superimposition of a linear magnetic field gradient on the original field introduces a spatial dimension and nuclei (here ${ }^{1} \mathrm{H}$ ), chemically equivalent, but physically separated along the gradient, will resonate at a frequency governed by the strength of the gradient and their respective positions. By applying three orthogonal field gradients it is possible to generate a three dimensional map of the distribution of the protons. The density of ${ }^{1} \mathrm{H}$ 's, moderated by restraints imposed by their physical and chemical environment, dictates the intensity of the signal (Chudek \& Hunter, 1997). Image intensity and contrast are further dependent on image acquisition parameters, which can be varied to highlight different features within the sample.

MRM as a monitoring process has already proved an ideal non-destructive tool in the observation of insects and larvae (Goodman et al., 1995) and especially the observation of parasitoid infection (Chudek et al., 1996, 1998).

However, whilst developing MRM protocols to study the interactions between $D$. coccinellae and $C$. septempunctata apparent anomalies were found in some images. These were related to the diet of the host insect. Images of C. septempunctata fed on their essential food, pea aphids [Acyrthosiphon pisum (Harris)], differ considerably from those fed on a standard artificial medium (Henderson \& Albrecht, 1988). By combining the MRM results with others obtained using more conventional ${ }^{1} \mathrm{H}$ NMR spectroscopy and $T_{1}$ and $T_{2}$ relaxation measurements it was possible to explain the apparent anomalies.

This paper describes the differences seen in the MRM images and attempts to explain these. It also shows how the presence of a $D$. coccinellae larva can be observed within the body of a live C. septempunctata host.

\section{METHODS}

\section{Sample preparation}

Insects. C. septempunctata were laboratory bred in large insect rearing cages and after eclosion they were fed pea aphids for 2 weeks prior to preparation for the experiments.

Feeding. C. septempunctata were fed on a diet of either $A$. pisum or an artificial medium the ingredients of which are shown in Table 1, for a period of five days before imaging.

Parasitisation. An aphid fed female C. septempunctata was placed with a female $D$. coccinellae. When four vigorous ovipositional attacks were observed the ladybird was considered infected and maintained, with food, in isolation for fourteen days until ready for imaging.

Imaging. A ladybird was placed without restraint in the bottom of a $10 \mathrm{~mm}$ outer diameter NMR tube. The tube was filled with $\mathrm{CO}_{2}$ gas from a generator and sealed with a plastic cap. Under these conditions the insect could be kept comatose for up to eighteen hours without any apparent physical harm.

\section{Analytical}

In all NMR spectroscopy and microimaging experiments a minimum of five replicates were used.

Spectroscopy. Proton NMR spectra were obtained for two groups of female ladybirds. Group 1 was fed pea aphids and Group 2 was fed an artificial diet.

Spectra were recorded on a Bruker AM 200 FT NMR spectrometer, with a $50 \mathrm{~mm}$ vertical bore superconducting magnet producing a magnetic field of 4.5 Tesla operating at a resonance frequency of $200.13 \mathrm{MHz}$ for protons. The sample was held in a $10 \mathrm{~mm}$ NMR tube and spectra were recorded using a $10 \mathrm{~mm}$ multinuclear probe tuned to ${ }^{1} \mathrm{H}$.

Imaging. Images of aphid and artificially fed female ladybirds were obtained using a Bruker AM 300WB spectrometer with an $89 \mathrm{~mm}$ vertical bore superconducting magnet producing a field of 7.1 Tesla operated at a resonance frequency of $300.13 \mathrm{MHz}$ for proton. This was fitted with a Bruker microimaging accessory with a $15 \mathrm{~mm}$ resonator.

Raw 3D data were generated using a standard Bruker 3D spin-echo pulse sequence with a chemical shift selective "soft" precursory pulse to excite water signals at $4.5 \mathrm{ppm}$, or lipid signals at $1.2 \mathrm{ppm}$. A matrix of $128 \times 128 \times 128$ data elements was collected and Fourier transformed on a Bruker X32 workstation using UXNMR. The resulting images are displayed as either maximum intensity projections or as surface reconstruction images. A maximum intensity projection is displayed as a pseudo 3D projection in which tissues showing apparent high proton density appear white, low proton densities as transparent and intermediate densities as different levels of translucency. A surface reconstruction image is where only certain apparent densities are displayed highlighting organs or features.

Relaxation measurements. $T_{1}$ and $T_{2}$ relaxation data were obtained for aphid-fed and artificially-fed female ladybirds on a Bruker AM300 FT NMR using Bruker inversion-recovery sequence for $T_{1}$ data, and a spin-echo program sequence for $T_{2}$ data.

\section{RESULTS}

Fig. 1 shows typical ${ }^{1} \mathrm{H}$ spectra of individual samples of C. septempunctata fed on (A) pea aphids or (B) the artificial medium. As can be seen the spectra differ considerably. Spectrum A shows two sharp resonances, the one at $\sim 4.5 \mathrm{ppm}$ can be assigned to the protons in water, the other at $\sim 1.3 \mathrm{ppm}$ to lipid protons. Spectrum B however shows one very broad resonance with several weak shoul-

TABLE 1. Ingredients of artificial food fed to C. septempunctata

\begin{tabular}{cc}
$6 \mathrm{~g}$ & ground dried yeast \\
$9 \mathrm{~g}$ & desiccated powdered liver \\
$15 \mathrm{~g}$ & sucrose \\
$10 \mathrm{ml}$ & maple syrup \\
$150 \mathrm{ml}$ & $\mathrm{H}_{2} \mathrm{O}$ \\
2 & powdered copper free vitamin pills \\
$2 \mathrm{~g}$ & agar \\
\hline
\end{tabular}




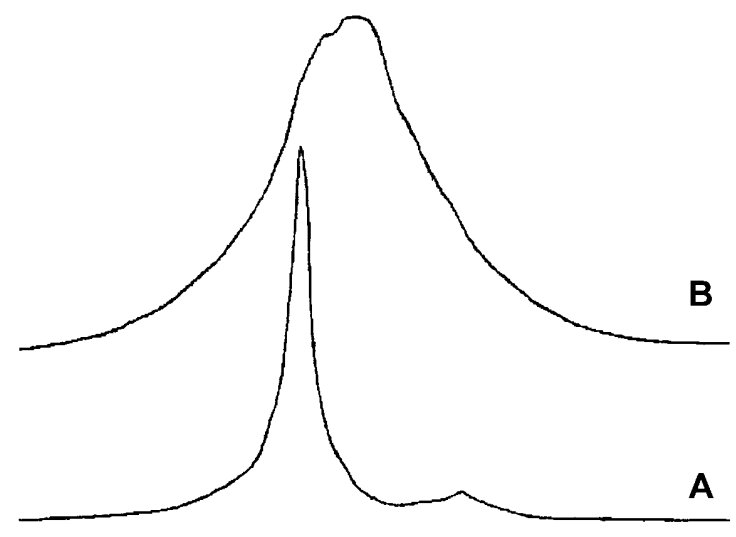

$\begin{array}{lllllllllllllll}10.0 & 9.0 & 8.0 & 7.0 & 6.0 & 5.0 & 4.0 & 3.0 & 2.0 & 1.0 & 0.0 & -1.0 & -2.0 & -3.0 & -4.0\end{array}$

Fig. 1. $200 \mathrm{MHz}$ proton spectra of live samples of C. septempunctata fed for 5 days before analysis with; A - aphids ( $A$. pisum); $\mathrm{B}$ - a standard artificial diet.

ders. It is impossible to assign these to any specific moiety.

The effects of diet are also clearly seen in the magnetic resonance microimages of C. septempunctata (Figs 2, A-D). In Figs 2, A-B, lateral and vertical views of the maximum intensity projection of data from an aphid fed ladybird, both the gut and ovaries can be clearly observed, along with what appears to be globules of fat spread through the rest of the abdomen. The equivalent projections of data from a ladybird fed on an artificial diet are completely different (Figs 2, C-D). Neither the gut nor the ovaries can be seen. The region of the abdomen exhibiting high proton density as seen in Figs 2, C-D is greater than that seen in Figs 2, A-B. Furthermore in Figs $2, \mathrm{C}-\mathrm{D}$ a region of very high proton density can be observed in the middle of the abdomen sited above where the gut is seen to run in aphid fed ladybirds (Figs 2, A-B). This difference is highlighted in the two surface reconstructions (Figs 2, G-H). The grey levels used to build the projections were chosen from the brightest in each data set. In Fig. $2 \mathrm{G}$ the gut and ovaries are clearly visible whereas in Fig. 2H they cannot be seen at all. Comparison of the two main NMR relaxation rates (measurements of the time required for excited nuclei to relax back to equilibrium) calculated for ladybirds fed on aphids and artificial diets (Table 2) show that there is an increase in the spin-lattice relaxation time, $T_{1}$, and a decrease in the spin-spin relaxation time, $T_{2}$.

Comparison of Figs 2, A-B with E-F is also interesting. In addition to the areas of higher proton den-

TABLE 2. NMR relaxation times of C. septempunctata sity corresponding to the guts and ovaries, other regions of higher proton density can be observed (Figs 2, E-F). An area to the front of the abdomen corresponds to the flight muscles whilst organs in the head, such as the eyes, can be seen.

After infection of $C$. septempunctata by the parasitic wasp, $D$. coccinellae, the MRM images change yet again. Figs 3, A-B show transverse and lateral views of an infected ladybird respectively. As well as the internal organs seen in aphid fed ladybirds (Figs 2, A-B, E-F), there is another area of higher intensity (circled, Roi 1) sited above and at an angle to the gut. This is likely to be an image of the parasitoid (which dissection of the host found in that region). Fig. $3 \mathrm{C}$ a view looking diagonally up through the ladybird shows the parasitoid larva clearly. Interestingly there only appears to be one ovary (shown in Roi 2). Roi 3 circles the region where the second ovary would be expected to be. Unfortunately it was not possible to ascertain if the host had two ovaries prior to infection, nor was a dissection carried out to confirm the loss.

Fig. 3D is a surface reconstruction of the parasitoid and the single ovary to the same scale and in the same orientation as the image in Fig. 3B.

\section{DISCUSSION}

The differences seen in the two NMR spectra (Figs 1, A-B) are striking, they show that the diet must have a major influence on the nature of the body tissues in C. septempunctata. Postulating the exact meaning of these differences is problematical. Spectra of aphid fed ladybirds show that the main body contents are water and lipid. While this can be assumed to also be the case with ladybirds fed on the artificial diet, the proton NMR spectra cannot be used to corroborate this. If the broad resonance seen in Fig. 1B was the result of broadening lines this would be reflected in a reduction in $\mathrm{T}_{2}$ (Table 2) (the half height line width of an NMR resonance is inversely proportional to $T_{2}$ ) (Abraham et al., 1990) and indeed this is the case. This has implications on the nature of the physical state or the environment of the protons to which the resonances relate and will be discussed further, below.

The images of the ladybirds show that the proton distribution, water and lipid also change as diet is changed. A build up of nutrient in some form could be explained by the mass of high proton density in the upper abdomen of artificially fed ladybirds. This does not take place in the aphid fed insects. Again examination of the relaxation times $T_{1}$ and $T_{2}$ may shed light on the problem. Many factors influence relaxation rates in the NMR experiment,

Aphid-fed ladybirds
$\mathrm{T}_{1}\left(\mathrm{H}_{2} \mathrm{O}\right)=0.8+/-0.2 \mathrm{~s}$
$\mathrm{T}_{1}$ (lipid) $=0.5+/-0.1 \mathrm{~s}$
$\mathrm{T}_{2}($ overall $)=0.008+/-0.002 \mathrm{~s}$

Artificially-fed ladybirds

$\mathrm{T}_{1}\left(\mathrm{H}_{2} \mathrm{O}\right)=1.4+/-0.1 \mathrm{~s}$

$\mathrm{T}_{1}($ lipid $)=1.0+/-0.3 \mathrm{~s}$

$\mathrm{T}_{2}($ overall $)=0.004+/-0.002 \mathrm{~s}$ 
A

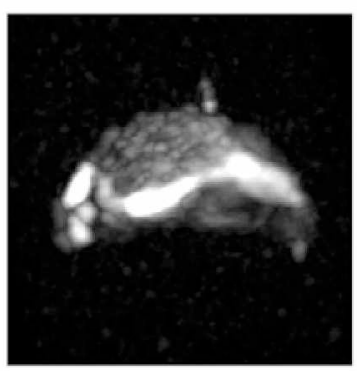

E

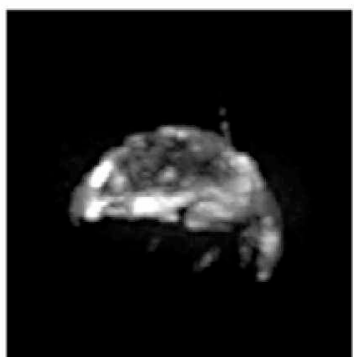

B

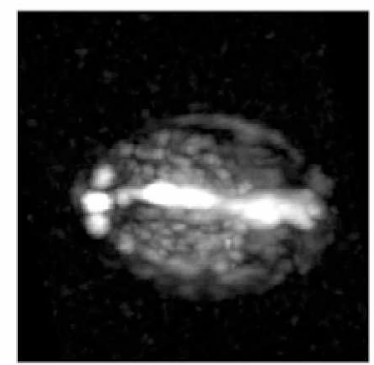

F

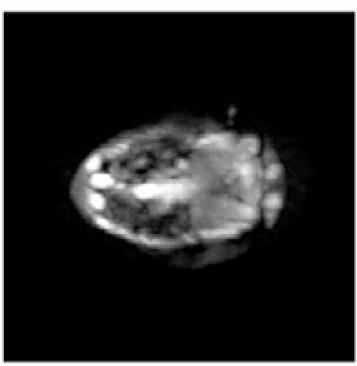

C

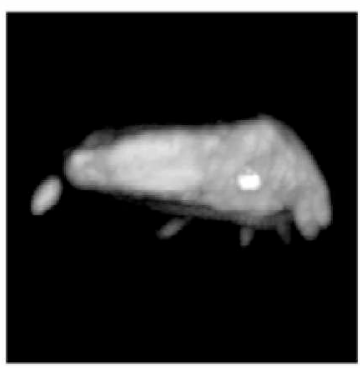

G

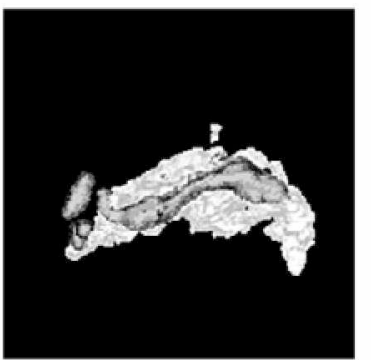

D

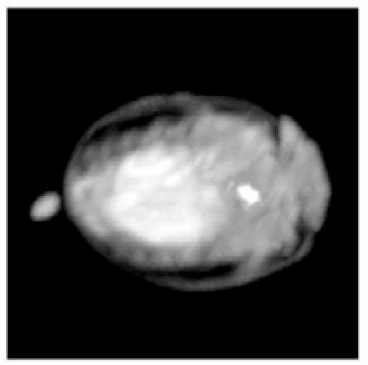

$\mathrm{H}$

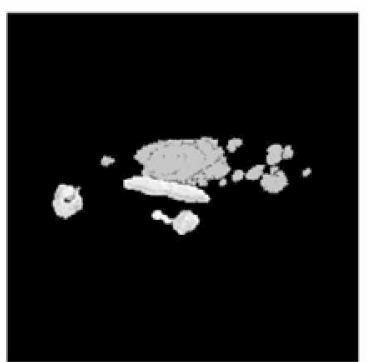

Fig. 2. A - lateral and B - vertical views of a maximum intensity projection of MRM data from the lipid protons of a live sample of $C$. septempunctata fed for 5 days before analysis with aphids $(A$. pisum $)$; $\mathrm{C}$ - lateral and $\mathrm{D}$ - vertical views of a maximum intensity projection of MRM data from the protons of a live sample of $C$. septempunctata fed for 5 days before analysis with a standard artificial diet; $\mathrm{E}$ - lateral and $\mathrm{F}$ - vertical views of a maximum intensity projection of MRM data from the water protons of a live sample of $C$. septempunctata fed for 5 days before analysis with aphids (A. pisum); $\mathrm{G}$ - aphid fed and $\mathrm{H}$ - artificial fed coccinellids; surface reconstructions of the voxels of highest proton density from the data sets used for $\mathrm{A}, \mathrm{B}$ and $\mathrm{C}, \mathrm{D}$ respectively.

one of the main factors being the rate of intra-and intermolecular motion. The first is chain motion, the second molecular movements such as Brownian motion. As these rates reduce $T_{1}$ begins to increase while $T_{2}$ decreases, as is seen in Table 2. This difference implies that the protons

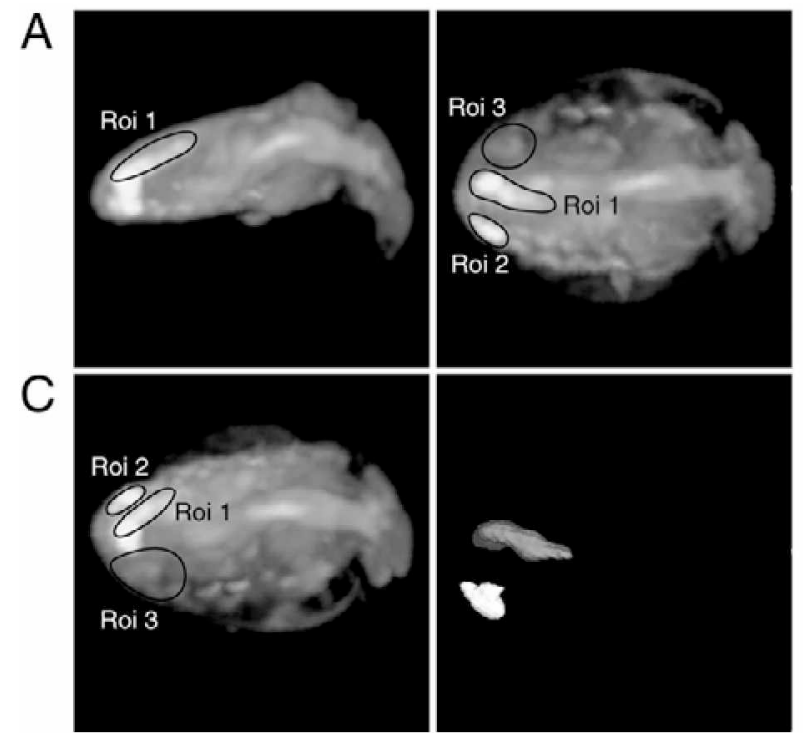

Fig. 3. A maximum intensity projection of a live sample of $C$ septempunctata infected by $D$. coccinellae viewed: A - lateral; $\mathrm{B}$ - vertical and C - oblique from below, highlighting Roi 1 a parasitoid; Roi 2 an ovary and Roi 3 the region where an ovary might be expected to be; and $\mathrm{D}$ - a surface reconstruction at the same scale and orientation as image $\mathrm{C}$ of the parasitoid and single ovary. in the artificially fed ladybirds are less mobile than those in the aphid fed ones. An explanation for the differences seen in the spectra, the images and the relaxation rates is that artificially fed ladybirds have laid down stocks of nutrients. This is possible when, in the absence of plentiful aphids, a time of limited sustenance is approaching, as for example in the prelude to diapause.

Samples of $C$. septempunctata infected by $D$. coccinellae also show changes. Most importantly the presence of the parasitoid larva can be observed within the host body. This will allow the host-parasitoid interactions to be monitored over the period of infection; it also opens the possibility of studying in real time the effects of superparasitism in insects.

\section{CONCLUSIONS}

Nuclear magnetic resonance spectroscopy and microimaging can be a powerful tool not only in the observation of changes in the anatomy of insects but in the nature of their tissues and body contents. It is also capable of observing a parasitoid larva within the body of the host insect. These observations can be made non-destructively on live insects allowing changes taking place within the insect to be monitored over an extended period.

ACKNOWLEDGEMENTS. Part of this work was carried out through funding from the Scottish Executive Rural Affairs Department. 


\section{REFERENCES}

Abraham R.J., Fisher J. \& LofTus P. 1990: Introduction to NMR Spectroscopy. John Willey \& Sons, Chichester.

Ceryngier P. \& Hodek I. 1996: Enemies of Coccinellidae. In Hodek I. \& Honěk A.: Ecology of Coccinellidae., Kluwer Academic Publishers, Dordrecht, pp. 319-350.

CHUdeK J.A. \& HUNTER G. 1997: Magnetic resonance imaging in plants. In Emsley J.W. \& Feeney J. (eds): Progress in N.M.R. Spectroscopy. Pergmon 31: 43-63.

Chudek J.A., Crook A.M.E., Hubbard S.F. \& Hunter G. 1996: Nuclear magnetic resonance microscopy of the development of the parasitoid wasp Venturia canescens within its host moth Plodia interpunctella. Magn. Reson. Imaging 14: 679-686.

Chudek J.A., Geoghegan I.E., Hunter G., MacKay R.L., Majerus M.E.N., Moritz S., McNicol R.J. \& Birch A.N.E. 1998: MRM, an alternative approach to the study of host/parasitoid relationships in insects. In Blumich B., Blumler P., Botto R. \& Fukushima E. (eds): Spatially Resolved Magnetic Resonance: Methods and Applications in Material Science, Agriculture and Biomedicine. Wiley-Uch., Weinheim, pp. 467-472.

Geoghegan I.E., Thomas W.P. \& Majerus M.E.N. 1997: Notes on the coccinellid parasitoid Dinocampus coccinellae (Schrank) (Hymenoptera: Braconidae) in Scotland. Entomologist 116: 179-184.
Goodman B.A., Gordon S.C., Chudek J.A., Hunter G. \& WOODFORD J.A.T. 1995: NMR microscopy as a non-invasive tool to study the development of Lepidopteran pupae. $J$. Insect Physiol. 41: 419-424.

Henderson S.A. \& Albrecht J.S. 1988: An artificial diet for maintaining ladybirds. Entomol. Rec. J. Var. 100: 261-264.

IPERTI G. 1964: Les parasites des Coccinelles aphidiphages dans les Basses-Alpes et les Alpes-Maritimes. Entomophaga 9: 153-180.

Klausnitzer B. 1969: Zur Kenntnis der Entomoparasiten mitteleuropäischer Coccinellidae. Abh. Ber. Naturh. Mus. Görlitz. 44: 1-15.

Kunn W. 1990: NMR microscopy - fundamentals, limits and possible applications. Angew. Chem. Int. Ed. Engl. 29: 1-19.

LuCK R.F. 1990: Evaluation of natural enemies for biological control: A behavioural approach. TREE 5: 196-199.

MAJERUS M.E.N. 1994: Ladybirds (New Naturalist 81). HarperCollins, London.

Majerus M.E.N. 1997: Parasitization of British ladybirds by Dinocampus coccinellae (Schrank) (Hymenoptera: Braconidae) Br. J. Entomol. Nat. Hist. 10: 15-24.

Miller D. 1971: Common Insects in New Zealand. A.H. \& A.W. Reed, Wellington.

Received January 6, 2000; accepted June 30, 2000 